\title{
Entropy-based measure of structural order in water
}

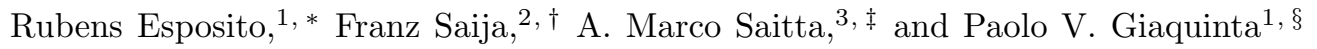 \\ ${ }^{1}$ Università degli Studi di Messina, \\ Dipartimento di Fisica, Contrada Papardo, 98166 Messina, Italy \\ ${ }^{2}$ CNR - Istituto per i Processi Chimico-Fisici, Sezione di Messina, \\ Via La Farina 237, 98123 Messina, Italy \\ ${ }^{3}$ Département de Physique des Milieux Denses, IMPMC, CNRS-UMR 7590, \\ B115, Université Pierre et Marie Curie, F-75252 Paris, France
}

(Dated: June 28, 2018)

\begin{abstract}
We analyze the nature of the structural order established in liquid TIP4P water in the framework provided by the multi-particle correlation expansion of the statistical entropy. Different regimes are mapped onto the phase diagram of the model upon resolving the pair entropy into its translational and orientational components. These parameters are used to quantify the relative amounts of positional and angular order in a given thermodynamic state, thus allowing a structurally unbiased definition of low-density and high-density water. As a result, the structurally anomalous region within which both types of order are simultaneously disrupted by an increase of pressure at constant temperature is clearly identified through extensive molecular-dynamics simulations.
\end{abstract}

PACS numbers: 05.20.Jj, 61.20.Ja, 64.10.+h, 64.70.Ja

Keywords: water, phase diagram, structural order, TIP4P, entropy, pair correlations

Water undergoes, under compression, a continuous structural transformation from an open, hydrogenbonded tetrahedral structure to a denser form in which the local molecular arrangement beyond the first coordination shell looks deeply modified [1]. Such two forms are ordinarily referred to as low-density and highdensity water (LDW, HDW). There is wide consensus on their being high-temperature manifestations of two glassy polymorphs of ice: low-density and high-density amorphous ice, respectively [2]. Raman and Brillouin spectroscopic studies have recently given conflicting predictions on the thermodynamic boundary between LDW and HDW forms 3, 4]. In this Letter we present an approach to analyze different ordering regimes in water that is entirely based on entropy and which can be used to trace the LDW-HDW boundary without resorting to any a priori assumptions on the local structure of the stable liquid phase.

Quantifying the degree of structural order present in liquids and amorphous materials is a challenging issue that has received considerable attention in the last few years [5, 6, 7, 8]. The possibility of characterizing in a synthetic way the macroscopic state of a non-crystalline substance through some integrated measure of the overall amount of order emerging from microscopic correlations has been promisingly exploited also for complex molecular liquids such as water [9, 10, 11]. The method is essentially based on the identification of appropriate metrics for both positional and angular order. The thermodynamic states of the system can then be mapped onto a structural diagram, spanned by such two parameters, which can be used to obtain a direct and quantitative insight into different ordering regimes achieved by materials under cooling and/or compression. In this Letter we introduce and analyze a metric for discussing struc- tural anomalies in water that is entirely and consistently based, for both translational and bond-orientational order, on the "fine structure" of the configurational entropy resolved through spatial correlations of increasing order.

The basic relation between entropy and order can be cast in a formally rigorous statistical-mechanical framework by resorting to the multi-particle correlation expansion of the configurational entropy 12, 13. For a classical, homogeneous and isotropic fluid, this expansion has the form:

$$
s_{\mathrm{ex}}=\sum_{n=2}^{\infty} s_{n},
$$

where $s_{\text {ex }}$ is the excess entropy per particle in units of the Boltzmann constant and the partial entropies $s_{n}$ are obtained from the integrated contributions of spatial correlations between $n$-tuples of particles. The pair entropy of a molecular fluid can be written as 14, 15]:

$$
s_{2}=-\frac{1}{2} \frac{\rho}{\Omega^{2}} \int\left\{g\left(\mathbf{r}, \omega^{2}\right) \ln \left[g\left(\mathbf{r}, \omega^{2}\right)\right]-g\left(\mathbf{r}, \omega^{2}\right)+1\right\} \mathrm{d} \mathbf{r} \mathrm{d} \omega^{2},
$$

where $\rho$ is the number density and $g\left(\mathbf{r}, \omega^{2}\right)$ is the pair distribution function (PDF) which depends on the relative separation $\mathbf{r}$ between a pair of molecules and on the set of Euler angles $\omega^{2} \equiv\left[\omega_{1}, \omega_{2}\right]$ that specify the relative orientations of the two particles in the laboratory reference frame. The quantity $\Omega$ represents the integral over the Euler angles of one molecule $\left(\Omega=8 \pi^{2}\right.$ for non-linear molecules). The pair entropy typically accounts for the overwhelming contribution to the total excess entropy of a dense liquid. In order to highlight the relative contributions of translational and orientational correlations

$$
s_{2}=s_{2}^{(\mathrm{tr})}+s_{2}^{(\mathrm{or})}
$$


we follow Lazaridis and Karplus [15] and factorize the $\mathrm{PDF}$ as

$$
g\left(r, \omega^{2}\right)=g(r) g\left(\omega^{2} \mid r\right) .
$$

In Eq. 4 $g(r)$ is the radial distribution function (RDF) for some arbitrary site in the molecules which, in the case of water, is identified with the oxygen atom. This function is obtained upon integration of the PDF over its angular coordinates. Instead, the function $g\left(\omega^{2} \mid r\right)$ represents the conditional distribution function for the relative orientation of a pair of molecules whose relative distance is $r$. This quantity is normalized to $\Omega^{2}$ and is referred to as the orientational distribution function (ODF). Using Eq. 4 one obtains for the translational and orientational contributions to the pair entropy:

$$
s_{2}^{(\operatorname{tr})}=-\frac{1}{2} \rho \int[g(r) \ln g(r)-g(r)+1] \mathrm{d} \mathbf{r},
$$

and

$$
s_{2}^{(\text {or })}=\rho \int g(r) S(r) \mathrm{d} \mathbf{r}
$$

with

$$
S(r)=-\frac{1}{2} \frac{1}{\Omega^{2}} \int g\left(\omega^{2} \mid r\right) \ln \left[g\left(\omega^{2} \mid r\right)\right] \mathrm{d} \omega^{2} .
$$

The use of the positive-definite quantity $-s_{2}^{(\operatorname{tr})}$ as a measure of translational order in both atomic and molecular fluids has been already suggested by Truskett and coworkers [ [6] and by Errington and Debenedetti [9] as an alternative to the integral of the absolute value of the total correlation function, $|g(r)-1|$, over a finite number of coordination shells. As requested for an order parameter, both quantities vanish in an ideal gas. As for the orientational order, the metric exploited so far for water is actually a measure of the degree of tetrahedrality in the average distribution of the four oxygen atoms that are closest to a given central atom 9, 10, 11, 16. As such, the definition of this order parameter rests on the knowledge of a pre-defined local structure. We propose, instead, to adopt $-s_{2}^{\text {(or) }}$ as an independent measure of angular order, au pair with $-s_{2}^{(\mathrm{tr})}$ for positional order. This mutually consistent choice for the translational and orientational metrics is rooted on a general and rigorous statistical-mechanical ground. As such, it can be implemented for any molecular fluid, without any a priori cognition of the local environment.

We calculated the translational and orientational pair entropies for the TIP4P model of liquid water [17]. Although more and more sophisticated classical potentials are still being developed [18, 19, 20, 21, 22, 23], the TIP4P description is, on the overall, very satisfactory when compared with experiments 24, 25], and its predictive power is well established 26, 27].
We carried out molecular-dynamics (MD) calculations, implemented through the PINY code 28] on a system of 108 water molecules in a cubic supercell with periodic boundary conditions. The sensitivity of the results to the size of the sample was tested with a number of simulations performed at ambient pressure on a system of 512 molecules. Long-range forces were computed through a particle-mesh Ewald method. The distance cutoff was set at $8.5 \AA$. The TIP4P model is based on a rigid-molecule description, in that it neglects intramolecular degrees of freedom. A time step of 2.5 fs turns out to be sufficient to ensure a proper dynamical evolution. Simulation times were in the $2.0 \mathrm{~ns}$ range. The configurations were stored every $1-2 \mathrm{ps}$. The resulting RDFs were calculated over 1000-2000 different configurations with a spatial resolution $\Delta r=0.05 \AA$. The ODF was calculated at intervals of $10^{\circ}$. We performed independent simulations in the NPT ensemble for pressures comprised between ambient pressure and $10 \mathrm{kbar}$ in the $210-400 \mathrm{~K}$ temperature range, with a step of $10 \mathrm{~K}$. Lower-density states $\left(0.95,0.90,0.85,0.80,0.75 \mathrm{~g} / \mathrm{cm}^{3}\right)$, corresponding to negative pressures, were investigated with constant-volume simulations carried out over the same temperature range. The initial set of positions and velocities of each run was taken from the last configuration of a simulation carried out at the same pressure, at an immediately higher or lower temperature.

At variance with $s_{2}^{(\mathrm{tr})}$, the calculation of $s_{2}^{(\mathrm{or})}$ poses severe difficulties. Indeed, computing and integrating the ODF - which, in principle, depends on up to nine independent variables - directly for a polyatomic molecular fluid over an extended range of temperatures and pressures is a formidable task. In practice, in the case of water, the $\mathrm{ODF}$ is a function of six variables only: i.e., the intermolecular distance, the two angles formed by the dipole vector of each molecule with the intermolecular axis, the angle describing the rotation of the two molecules around the intermolecular axis, and the two angles describing the rotation of each water molecule around its dipole vector. In order to tackle this fairly demanding computational task, we resorted to an approximate mapping of the ODF onto its low-density form. This mapping, known as "adjusted gas-phase" (AGP) approximation, was introduced by Lazaridis and Karplus [15] and is implemented under a two-fold constraint: i) the requirement that the "exact" - i.e., numerically evaluated - orientationally averaged interaction energy between two water molecules be reproduced, as a function of their relative distance, in the liquid phase; ii) the consistency of the approximate ODF with the oneand two-dimensional projections of the exact function over the five-dimensional space spanned by the angular coordinates. The reliability of the AGP approximation has been positively checked through a number of indicators such as the oxygen-oxygen and oxygen-hydrogen site-site distribution functions. An independent direct 


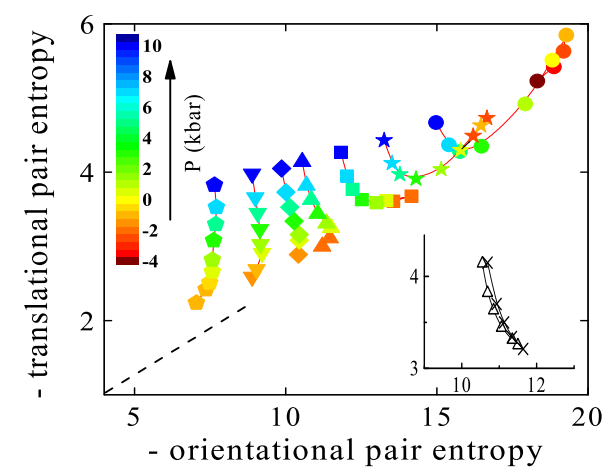

FIG. 1: (Color online) Ordering map: Translational order parameter, $-s_{2}^{(\mathrm{tr})}$, plotted as a function of the orientational order parameter, $-s_{2}^{\text {(or) }}$, for 108 particles at different temperatures (entropy units: cal $/ \mathrm{mol} \mathrm{K}$ ). Circles, $T=210 \mathrm{~K}$; stars, $T=240 \mathrm{~K}$; squares, $T=270 \mathrm{~K}$; upward triangles, $T=300 \mathrm{~K}$; diamonds, $T=320 \mathrm{~K}$; downward triangles, $T=350 \mathrm{~K}$; pentagons, $T=400 \mathrm{~K}$. Markers are colored according to the pressure of the liquid. For $T \geq 300 \mathrm{~K}$, increasing pressures lead to increasing values of $-s_{2}^{(\mathrm{tr})}$; for $T<300 \mathrm{~K}$, increasing pressures first produce an increase of both order parameters (negative-pressure states), followed by their simultaneous decrease that partially stops at the minimum of the ordering locus beyond which the translational order parameter starts growing again. The dotted line has vanishing intercept (not shown) and was traced as a guide for the eye to follow the asymptotic behavior of the order parameters at high temperatures and low pressures. The inset shows a comparison between the results obtained at $T=300 \mathrm{~K}$ with 108 (triangles) and 512 particles (crosses).

calculation [? ] of the orientational pair entropy of 512 TIP4P water molecules carried out at constant volume $\left(\rho=0.999 \mathrm{~g} / \mathrm{cm}^{3}\right)$ at $T=298 \mathrm{~K}$ leads to a value $\left(-s_{2}^{(\text {or })}=50.08\right.$ Joule $\left./ \mathrm{mol} \mathrm{K},[30]\right)$ that is in acceptable agreement with the constant-pressure (1 atm) AGP estimates obtained with 216 molecules at the same temperature $\left(-s_{2}^{\text {(or) }}=48.74 \mathrm{Joule} / \mathrm{mol} \mathrm{K}, \underline{31}\right)$, and with 512 molecules at $T=300 \mathrm{~K}\left(-s_{2}^{\text {(or) }}=48.70 \mathrm{Joule} / \mathrm{mol} \mathrm{K}\right.$, this work). We note that the pair entropy already represents more than $80 \%$ of the total configurational entropy of TIP4P water at the highest temperature and largest pressure that we have investigated. Moreover, this fraction increases with decreasing temperatures and pressures until $-s_{2}$ eventually overcomes $-s_{\mathrm{ex}}$ [32]. On the other hand, orientational correlations account for the overwhelming contribution to the pair entropy. We found that this contribution also increases, with decreasing temperatures and pressures, from a percent value of $\sim 67 \%$ for $T=400 \mathrm{~K}$ and $P=10 \mathrm{kbar}$ to $\sim 79 \%$, about which it appears to saturate at low enough temperatures and pressures. We now turn to a discussion of

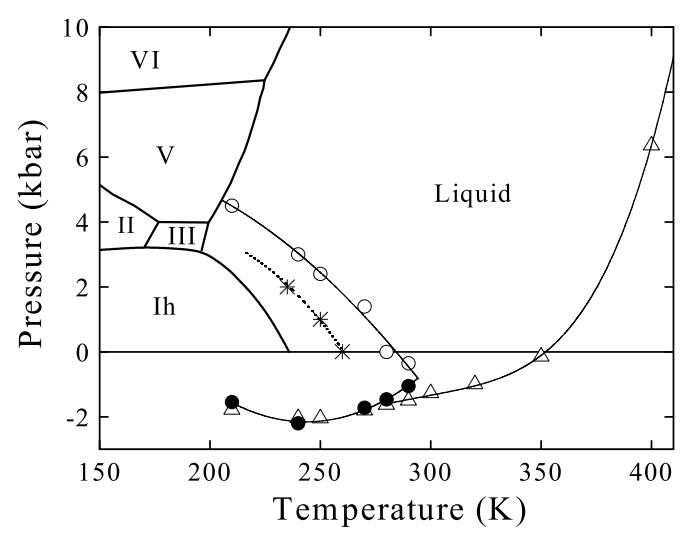

FIG. 2: Phase diagram of the TIP4P model for water: solid circles and open triangles identify states corresponding to the maxima observed in $-s_{2}^{(\operatorname{tr})}(\rho)$ and $-s_{2}^{(\text {or })}(\rho)$, respectively; open circles identify states corresponding to the minimum exhibited by $-s_{2}^{(\operatorname{tr})}(\rho)$. Stars locate the states of maximum liquid density. Lines traced across the data points are a guide for the eye. The solid-solid and solid-liquid boundaries are sketched after the coexistence lines shown in Fig. 1 of [23] for the TIP4P model.

the thermodynamic behavior of $-s_{2}^{(\operatorname{tr})}$ and $-s_{2}^{(\text {or })}$. The former quantity behaves differently according to temperature. For $T \geq 300 \mathrm{~K}$, the translational order parameter monotonically increases - over the explored range - as a function of density or pressure, as is typically observed in an ordinary simple fluid [8]. Below ambient temperature, this behavior is still maintained at either low or high enough pressures: for $T \lesssim 295 \mathrm{~K}$, a window actually opens up within which $-s_{2}^{(\mathrm{tr})}$ decreases with increasing pressures. As for the orientational order parameter, this quantity shows a maximum as a function of density, which sharpens with decreasing temperatures. For $T \lesssim 280 \mathrm{~K}$, the maximum observed in both quantities falls - to within the numerical uncertainty of the calculations - around the same density $\left(\sim 0.92 \mathrm{~g} / \mathrm{cm}^{3}\right)$,

The nature of the correlation between translational and orientational order can be enlightened upon constructing a two-parameter ordering map, as originally proposed by Torquato and coworkers in [5]. The map generated by the order parameters introduced in this Letter is shown in Fig. 1, where we plotted $-s_{2}^{(\operatorname{tr})}$ as a function of $-s_{2}^{\text {(or) }}$. We identify three distinct regimes according to the nature of the structural response of water to compression. At high temperatures, both translational and orientational order are enhanced by pressure in lowdensity water. However, at higher densities, a different structural condition develops, characterized by opposite behaviors of the two order parameters: in this hybrid regime the translational order keeps increasing while the 
orientational order decreases as the pressure is raised further and further. At low temperatures, the compression of the liquid may also weaken both types of order: such a twofold anomalous behavior is observed in an intermediate range of densities whose borders on the ordering locus coincide with either the relative or absolute maximum of $-s_{2}^{(\mathrm{or})}$ or $-s_{2}^{(\mathrm{tr})}$, respectively, and with the minimum of $-s_{2}^{(\operatorname{tr})}$. In passing, we note that the shape of the ordering map is not appreciably modified by the size of the calculation (see the inset in Fig. 1).

The mapping of the $(T, P)$ domains corresponding to the different ordering regimes outlined above onto the phase diagram of TIP4P water is shown in Fig. 2. The lower boundary of the structurally anomalous region is entirely located in the negative pressure range. This threshold shifts to higher pressures with increasing temperatures. However, it is interesting to note that this line traces, almost all over the range, a constant-density path $\left(\rho \simeq 0.92 \mathrm{~g} / \mathrm{cm}^{3}\right)$. We surmise that, in the light of the present results, this density can be interpreted as a sort of intrinsic threshold - in the liquid phase - for the nucleation of a stable solid with perfect tetrahedral co-ordination. Indeed, the density of the TIP4P Ih ice at ambient pressure $\left(0.937 \mathrm{~g} / \mathrm{cm}^{3}\right)$ 23] is consistent with this hypothesis. We also recall that the corresponding experimental value is $\sim 0.92 \mathrm{~g} / \mathrm{cm}^{3}$ [33].

The upper boundary of the anomalous region shifts to lower pressures with increasing temperatures. Correspondingly, the density decreases from $\sim 1.2 \mathrm{~g} / \mathrm{cm}^{3}$ to $\sim 0.95 \mathrm{~g} / \mathrm{cm}^{3}$. As seen from the figure, a twofold anomalous structural behavior is observed in the liquid coexisting with ices Ih, III, and, partially, V. It is rather natural to explain such an anomalous behavior as due to the presence of a persistent and extended hydrogen-bonded network. Consequently, we are lead to identify the associated region as the LDW structural basin, as opposed to the HDW basin where the translational and orientational order parameters show opposite trends as a function of the pressure. The lower boundary of the HDW region, separating above ambient temperature thermodynamic regions where the angular order is either enhanced or disrupted by pressure, crosses the $P=0$ axis at a temperature $(T \simeq 350 \mathrm{~K})$ that is close to the boiling point of TIP4P water at ambient pressure $(T \simeq 363 \mathrm{~K})[34$. We also note that the locus of density maxima lies entirely inside this region as also found by Errington and Debenedetti through a tetrahedricity-based order representation [9].

On the basis of the analysis of the experimental data carried out by Soper and Ricci [1], one is lead to identify the LDW-HDW crossover threshold with the state of the liquid whose oxygen-oxygen structure factor can be represented by an equal-weight superposition of the "asymptotic" LDW and HDW forms. At $T=268 \mathrm{~K}$, this criterion yields a pressure of $\sim 1 \mathrm{kbar}$. The esti- mate obtained with the present method for the TIP4P model at the same temperature is about $1.2 \mathrm{kbar}$. As for the thermodynamic slope of the LDW-HDW structural boundary, our results are consistent with the numerical predictions of Saitta and Datchi 27], who used a criterion based on bond-angles distributions to trace the crossover line, and with the experimental findings of $\mathrm{Li}$ and coworkers [4].

In this Letter we have implemented, on a rigorous statistical-mechanical basis, an entropy-based theoretical framework that allows a structurally unbiased description of ordering regimes in liquid water, in its thermodynamically stable domain. The current individuation of LDW and HDW basins is in good agreement with neutron-diffraction results at $268 \mathrm{~K}$ and further clarifies other conflicting experimental data. We plan to extend this analysis to the study of amorphous ices. However, the proposed metrics prospectively qualify the current method as a general tool for investigating the nature of structural order in molecular liquids.

We thank Dr. J. Zielkiewicz for providing us with his numerical estimate of the orientational pair entropy for the TIP4P model. One of the authors (R. E.) acknowledges the support of a Socrates/Erasmus grant and the scientific hospitality of the Université Pierre et Marie Curie during the Erasmus stage.

* Electronic address: rubens.re@libero.it

$\dagger$ Electronic address: saija@me.cnr.it

¥ Electronic address: marco.saitta@impmc.jussieu.fr

$\S$ Corresponding author; Electronic address: paolo.giaquinta@unime.it

[1] A. K. Soper and M. A. Ricci, Phys. Rev. Lett. 84 (2000) 2881

[2] O. Mishima and H. E. Stanley, Nature 396 (1998) 329

[3] T. Kawamoto, S. Ochiai, and H. Kagi, J. Chem. Phys. 120 (2004) 5867

[4] F. Li, Q. Cui, Z. He, T. Cui, J. Zhang, Q. Zhou, and G. Zou, J. Chem. Phys. 123 (2005) 174511

[5] S. Torquato, T. M. Truskett, and P. G. Debenedetti, Phys. Rev. Lett. 84 (2000) 2064

[6] T. M. Truskett, S. Torquato, and P. G. Debenedetti, Phys. Rev. Lett. 62 (2000) 993

[7] A. R. Kansal, S. Torquato, and F. H. Stillinger, Phys. Rev. E 66 (2002) 041109

[8] J. R. Errington, P. G. Debenedetti and S. Torquato, J. Chem. Phys. 118 (2003) 2256

[9] J. R. Errington and P. G. Debenedetti, Nature 409 (2001) 318

[10] R. M. Lynden-Bell and P. G. Debenedetti, J. Phys. Chem B 109 (2005) 6527

[11] N. Giovambattista, P. G. Debenedetti, F. Sciortino and H. E. Stanley, Phys. Rev. E 71 (2005) 061505

[12] H. S. Green, The Molecular Theory of Fluids, NorthHolland, Amsterdam 1952

[13] R. E. Nettleton and M. S. Green, J. Chem. Phys. 29 (1958) 1365 
[14] T. Lazaridis and M. E. Paulaitis, J. Phys. Chem. 96 (1992) 3847

[15] T. Lazaridis and M. Karplus, J. Chem. Phys. 105 (1996) 4294

[16] P. -L. Chau and A. J. Hardwick, Mol. Phys. 93 (1998) 511

[17] W. L. Jorgensen, J. Chandrasekhar, J. D. Madura, R. W. Impey and M. L. Klein, J. Chem. Phys. 79 (1983) 926

[18] W. L. Jorgensen and C. Jenson, J. Comp. Chem. 19 (1998) 1179

[19] M. W. Mahoney, and W. L. Jorgensen, J. Chem. Phys. 112 (2000) 8910

[20] B. Guillot and Y. Guissani, J. Chem. Phys. 114 (2001) 6720

[21] H. Nada and J. P. van der Eerden, J. Chem. Phys. 118 (2003) 7401

[22] C. Nieto-Draghi, J. Bonet Avalos and B. Rousseau, J. Chem. Phys. 118 (2003) 7954

[23] J. L. F. Abascal, E. Sanz, R. García-Fernández, and C. Vega, J. Chem. Phys. 122 (2005) 234511

[24] J. M. Sorenson, G. Hura, R. M. Glaeser and T. Head-
Gordon, J. Chem. Phys. 113 (2000) 9149

[25] A. M. Saitta, T. Strässle, G. Rousse, G. Hamel, S. Klotz, R. J. Nelmes and J. S. Loveday, J. Chem. Phys. 121 (2004) 8430

[26] O. Mishima, L. D. Calvert and E. Whalley, Nature 310 (1984) 393

[27] A. M. Saitta and F. Datchi, Phys. Rev. E (RC) 67 (2003) 020201

[28] G. J. Martyna, M. E. Tuckerman and M. L. Klein, PINY_MD (c) Simulation Package; Copyright (c)2002

[29] J. Zielkiewicz, J. Chem. Phys. 123104501 (2005); erratum 124 (2006) 109901

[30] J. Zielkiewicz, private communication

[31] T. Lazaridis, private communication

[32] F. Saija, A. M. Saitta and P. V. Giaquinta, J. Chem. Phys. 119 (2003) 3587

[33] V. F. Petrenko and R. W. Whitworth, Physics of Ice, Oxford University Press, Oxford (1999)

[34] M. J. Vlot, J. Huinink and J. P. van der Eerden, J. Chem. Phys. 110 (1999) 55 